\title{
Quantum Calculations of the Velocity Dependence of the Differential and Total Cross Sections for Elastic Scattering of Molecular Beams*
}

\author{
RichaRd B. BERNSTEIN \\ Chemistry Department, The University of Michigan, Ann Arbor, Micligan
}

(Received August 10, 1960)

\begin{abstract}
The partial wave treatment (phase shift analysis) of the elastic scattering of molecular beams [J. Chem. Phys. 33, 795 (1960) ] is applied to the calculation of the velocity dependence of the differential and total cross sections for an assumed L-J $(12,6)$ potential. For most of the calculations, the $\epsilon, \sigma$ values are chosen to correspond to the $\mathrm{H}_{2}-\mathrm{Hg}$ system. The range of the velocity parameter $A \equiv k \sigma=\mu \gamma \sigma / \hbar$ is from 3 to 30 ; this is equivalent to a 100 -fold variation in $\mathrm{H}_{2}$ beam temperature (approx $8.2-820^{\circ} \mathrm{K}$ ). Computations of the angular distribution of the scattering $d \sigma(\theta) / d \Omega$ and the total cross section $Q$ as a function of $A$ are reported. A correlation of the interference maxima in $d \sigma(\theta) / d \Omega$ is presented. The Massey-Mohr approximation for $Q(A)$ for an inverse sixth-power attractive potential is compared with the present calculations for the $\mathrm{L}-\mathrm{J}(12,6)$ potential. Significant undulatory deviations are noted at low $A$; this effect is attributed to the existence of the broad maximum in the phase shift curve $\eta(l)$, which, in turn, originates from the negative repulsive phases at low $l$. Consideration is given to the question of the sensitivity of the scattering to the repulsive part of the potential.
\end{abstract}

\section{INTRODUCTION}

$I^{1}$ $T$ is well-known that the classical treatment of elastic scattering ${ }^{\mathbf{1}, 2}$ is inapplicable under certain conditions, and in particular, yields no information regarding low-angle differential scattering or the total cross section. Massey and $\mathrm{Mohr}^{3}$ applied the quantummechanical (partial wave) method to the molecular scattering problem, obtaining an exact solution for the rigid-sphere model ${ }^{3 a}$ and an approximate one for a general inverse-power intermolecular potential..$^{3 \mathbf{b}}$ For the latter case a simple approximation for the total cross section $Q$ was derived; for the potential

$$
V(r)=-C / r^{6},
$$

the Massey-Mohr equation takes the form

$$
Q=b(C / v)^{2 / 5},
$$

where $v$ is the relative velocity and $b$ a known constant. The differential scattering cross section $d \sigma(\theta) d \Omega$ [alternatively written $I(\theta)$ ] could not be expressed in simple terms.

In a previous paper ${ }^{4}$ a partial wave treatment (phase shift analysis) was presented for the scattering of molecules subject to a Lennard-Jones $(12,6)$ potential,

$$
V(r)=4 \epsilon\left[(\sigma / r)^{12}-(\sigma / r)^{6}\right] .
$$

The repulsive part of the potential leads to negative phase shifts $\left(\eta_{l}\right)$ for the lower order partial waves and

* The author acknowledges with thanks financial support of this work from the U.S. Atomic Energy Commission, Division of Research, and from the Alfred P. Sloan Foundation.

1 J. F. Hirschfelder, C. F. Curtiss, and R. B. Bird, Molecular Theory of Gases and Liquids (John Wiley \& Sons, Inc., New York, 1954).

2 E. A. Mason, J. Chem. Phys. 26, 667 (1957)

${ }^{3}$ (a) H. S. W. Massey and C. B. O. Mohr, Proc. Roy. Soc. (London) A141, 434 (1933); (b) A144, 188 (1934).

* R. B. Bernstein, J. Chem. Phys. 33, 795 (1960), hereafter referred to as $I$. thus gives rise to a maximum in $\eta(l)$, whose location and value are dependent upon the velocity parameter

$$
A \equiv k \sigma=\mu \nu \sigma / \hbar \text {. }
$$

It is the purpose of the present paper to investigate the influence of the repulsion (via the phase shifts) upon the differential and total scattering cross sections and their velocity dependence. In this connection it has been of interest to investigate the applicability of the Born approximation and the validity of the MasseyMohr equation when applied to molecular scattering according to an L-J $(12,6)$ potential.

\section{PROCEDURE}

The symbols used are those of I. The two parameters found convenient to characterize the scattering system are

$$
A=k \sigma, \quad B=\epsilon \sigma^{2} 2 \mu / \hbar^{2},
$$

where $k=2 \pi / \lambda=\mu v / \hbar$ is the propagation number, $\mu$ the reduced mass, and $v$ is the (initial) relative velocity. For most of the calculations to be presented, the parameters $\epsilon$ and $\sigma$ have been chosen the same as in $\mathbf{I}$, to correspond fairly closely to the $\mathrm{H}_{2}-\mathrm{Hg}$ system: $\epsilon=$ $2.46 \times 10^{-14} \mathrm{erg}, \sigma=2.91 \mathrm{~A}$. Here $B=125$ and $A$ has been varied from 3 to 30 . This is equivalent to a $100-$ fold range in the $\mathrm{H}_{2}$ beam temperature (i.e., from 8.2 $820^{\circ} \mathrm{K}$, assuming (from I) the equivalence: $T=295^{\circ} \mathrm{K}$ at $A=18$ ) .

Phase shifts were taken from Table III of I, suitably extended to higher $l$ (always until $\eta_{l} \leqslant 0.03$ ) by means of the Born approximation (to be discussed below). On using the standard Mott-Massey ${ }^{5 a}$ equations, $I(\theta)$ and $Q$ were computed from the phases at $A=$ $3,5,7,9,10,14.1,15,18,20,24$, and 30 using an

${ }^{5}$ N. F. Mott and H. S. W. Massey, The Theory of Atomic Collisions (Clarendon Press, Oxford, 1949), 2nd ed.; (a) p. 24; (b) p. 118 . 


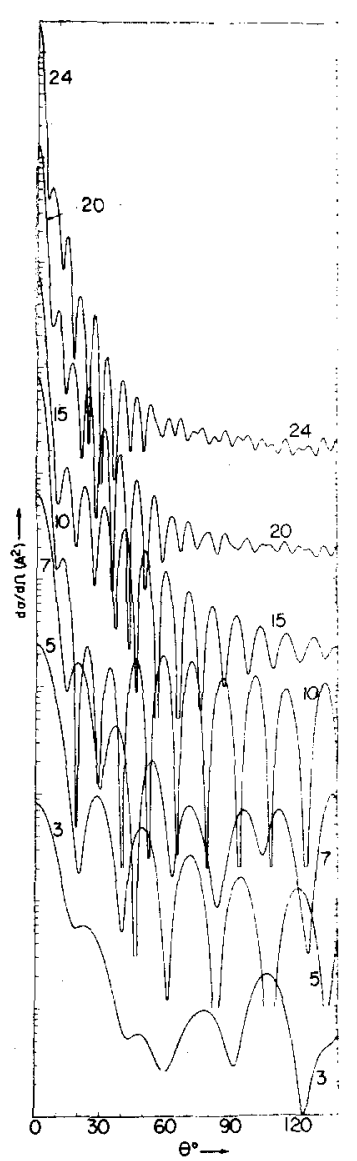

FIg. 1. Typical calculated angular distributions. Parameter: $A$. $B=125$ for all curves. Note that the vertical $(\log )$ scale has been shifted by one cycle progressively for each succeeding curve (see text).

IBM 704 computer. The program yielded $I(\theta)$ (in $\mathrm{A}^{2}$ ) and $I^{*}(\theta) \equiv I(\theta) / \pi \sigma^{2}$ from $0,(0.5), 180^{\circ}$ as well as $Q$ (in $\mathrm{A}^{2}$ ) and $Q^{*}=Q / \pi \sigma^{2}$. Computing time for one given value of $A$ varied from 0.5 to $1.5 \mathrm{~min}$ (in the latter case the calculation involved 120 phases).

\section{VELOCITY DEPENDENCE OF THE ANGULAR DISTRIBUTION}

Figure 1 shows some typical angular distributions. Here $A=3,5,7,10,15,20$, and $24 ; \theta$ extends to $140^{\circ}$. For clarity of presentation, the vertical (logarithmic) scale has been shifted by one cycle progressively for each succeeding curve. To fix the location of the curves, the values of the intercepts $\left[I(0)\right.$ in $\left.A^{2}\right]$ are listed, as follows,

$$
\begin{array}{cccccccc}
A & 3 & 5 & 7 & 10 & 15 & 20 & 24 \\
10^{-3} \times I(0)\left(\mathrm{A}_{2}\right) & 0.82 & 2.51 & 6.48 & 8.14 & 7.33 & 13.1 & 21.4 .
\end{array}
$$

The positions of the interference maxima and minima are correlated in Fig. $2 .{ }^{6}$ The index $N$ is an integer for a

\footnotetext{
${ }^{6}$ For $A \leq 10$ the undulations in $d \sigma(\theta) / d \Omega$ are noticeably less regular; this is seen (to a lesser extent) in Fig. 2 . As discussed in $\mathrm{I}$, for $B=125$ and $A \leq 10$ penetration of the centrifugal barrier occurs (classically, the condition for orbiting is $K \leq 0.8$ ) giving rise to one or more discontinuities in the $\eta(l)$ curves; this factor may be of some importance here.
}

maximum, half-integer for a minimum. As pointed out in I, the periodicity of the undulations in the angular distributions is governed primarily by $A$. This is seen quite clearly in Figs. 1 and 2.

It would be desirable to construct a unique "reduced scattering curve," i.e., a universal representation for the velocity-dependent angular distributions (Fig. 1). This cannot be expected, however, due to the complexity of the problem when the reduced relative kinetic energy

$$
K \equiv \frac{1}{2} \mu v^{2} / \epsilon=A^{2} / B
$$

is of order unity and the incident deBroglie wave is seriously distorted by the scattering potential. As pointed out in $\mathrm{I}$, this distortion is also dependent upon $A$ (or, equivalently, the ratio of the wavelength to the extension of the force field).

A step in this direction may be taken if one restricts attention to the scattering at low angles and at high $A$, where the wave is not much diffracted by the scattering field (conditions appropriate for the Born approximation; see the following). Here the undulations in the angular distribution $I(\theta)$ should be determined primarily ${ }^{5 b}$ by the variable $k s$, where

$$
s=2 \sin (\theta / 2),
$$

or, equivalently, by the dimensionless quantity

$$
k s \sigma=2 A \sin (\theta / 2) \text {. }
$$

Figure 3 is a plot with abscissa $2 A \sin (\theta / 2)$, for $A=$ $15,20,24$, and 30 . In the low-angle region particularly, the curves show similarity; the major part of the velocity dependence is thus fairly well correlated. For smaller velocities $(A \leq 10)$, the correlation is less satisfactory, as anticipated.

\section{RELATION BETWEEN $I(0)$ AND $Q$}

Massey and $\mathrm{Mohr}^{3 a}$ have considered the limiting case when a very large number of phase shifts are re-

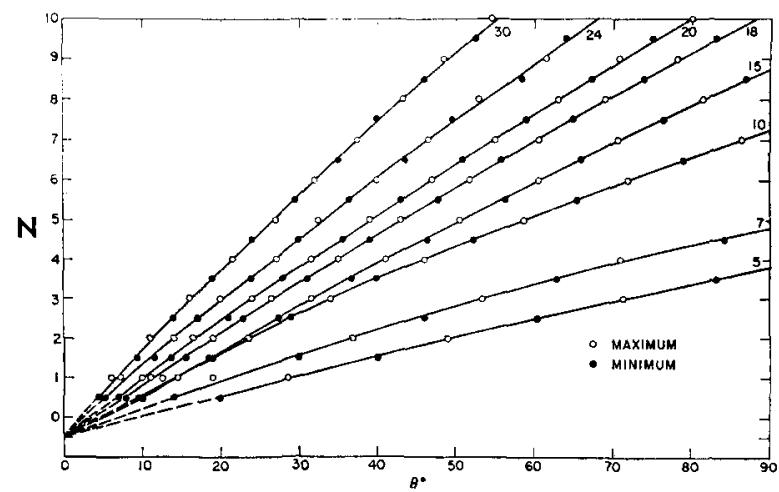

FIG. 2. Correlation of interference peaks in angular distributions. Parameter: $A . B=125$ for all curves. The index $N$ is an integer for a maximum, half-integer for a minimum.

${ }^{7}$ The ordinate is $\left(1 / A^{2}\right) d \sigma(\theta) / d \Omega$. The rationale for this choice is presented in the next section. 
quired in the calculation of $I(\theta)$, and the phases (after removing multiples of $\pi$ ) are essentially random numbers ( $\eta_{l}$ oscillating rapidly with $l$ ). Under these conditions one finds that

$$
I(0)=(k Q / 4 \pi)^{2} \text {. }
$$

For the "rigid-sphere" potential $Q$ is velocity independent; for a realistic intermolecular potential $Q$ is a relatively insensitive function of velocity (see discussion following). With the approximation of nearly constant $Q$, Eq. (9) implies $I(0) / k^{2} \cong$ constant. To remove the major velocity dependence of $I(0)$ the ordinate in Fig. 3 was therefore chosen to be $\left(1 / A^{2}\right) d \sigma / d \Omega$.

It is convenient to rewrite Eq. (9) in terms of the reduced quantities $I^{*}(\theta)$ and $Q^{*}$. Thus

$$
I^{*}(0) \cong\left(A Q^{*} / 4 \pi^{\frac{1}{2}}\right)^{2} \text {. }
$$

The ratio $j(A)$ is defined,

$$
j(A) \equiv I^{*}(0) /\left(A Q^{*} / 4 \pi^{3}\right)^{2},
$$

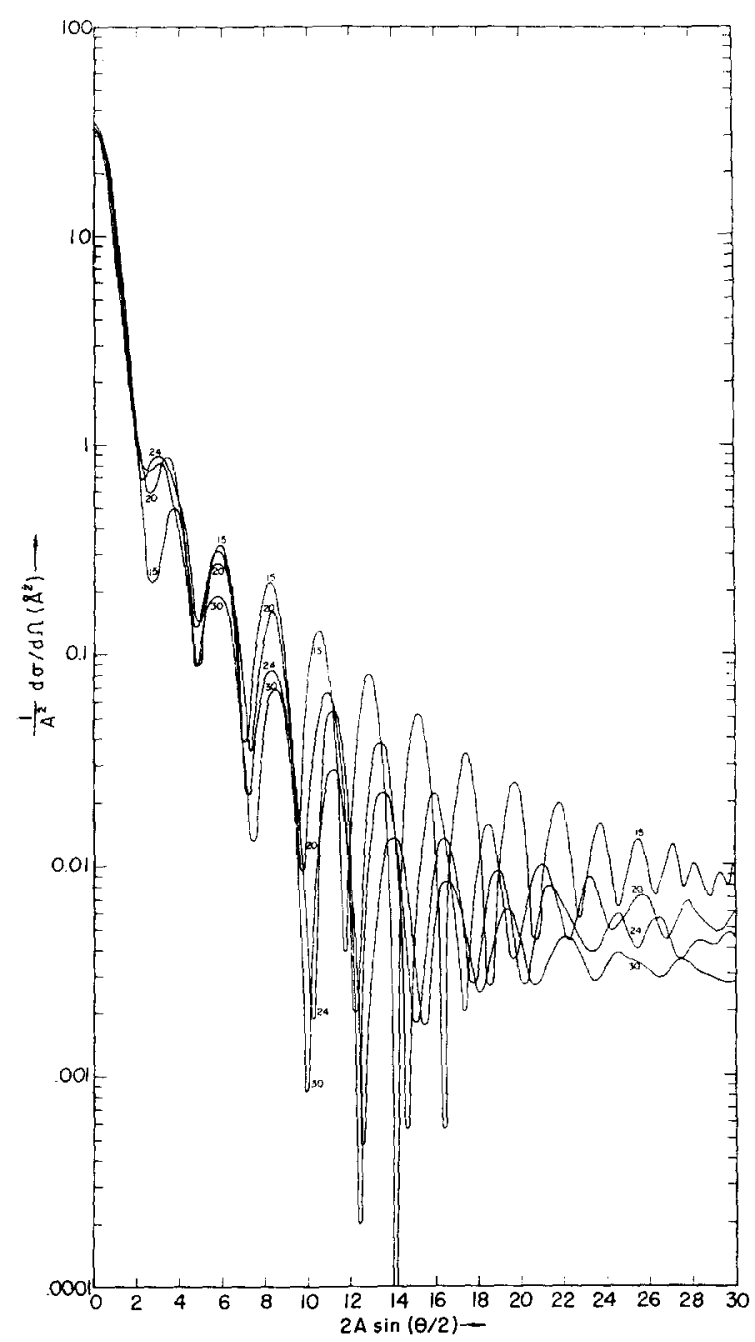

FIG. 3. Reduced scattering curves. Parameter: $A$. $B=125$ for

\begin{tabular}{|c|c|c|c|c|c|}
\hline \multirow[b]{2}{*}{$A$} & \multicolumn{4}{|c|}{ L.-J. $(12,6) ; B=125$} & \multirow{2}{*}{$\begin{array}{c}\text { Rigid } \\
\text { sphere } \\
j(\boldsymbol{A})\end{array}$} \\
\hline & $I^{*}(0)$ & $Q^{*}$ & $I^{*}(0) / A^{2}$ & $j(A)$ & \\
\hline 3 & 30.8 & 7.27 & 3.42 & 3.25 & 1.47 \\
\hline 5 & 94.3 & 13.17 & 3.77 & 1.09 & 1.26 \\
\hline 7 & 244 & 14.06 & 4.98 & 1.27 & \\
\hline 9 & 299 & 10.69 & 3.69 & 1.62 & \\
\hline 10 & 306 & 9.16 & 3.06 & 1.83 & 1.11 \\
\hline 14.1 & 245 & 6.58 & 1.23 & 1.43 & \\
\hline 15 & 275 & 6.67 & 1.22 & 1.38 & \\
\hline 18 & 395 & 7.16 & 1.22 & 1.20 & \\
\hline 20 & 491 & 7.41 & 1.23 & 1.12 & 1.05 \\
\hline 24 & 806 & 7.60 & 1.40 & 1.22 & \\
\hline 30 & 1208 & 7.08 & 1.34 & 1.35 & \\
\hline $40^{\mathrm{s}}$ & $(1750)$ & $(5.80)$ & $(1.09)$ & $(1.63)$ & \\
\hline
\end{tabular}
all curves.
TABLE I. Relation between $I^{*}(0)$ and $Q^{*}$.

"These computations less reliable than others.

which, for the rigid-sphere model at least, should approach unity in the high velocity limit.

Table I lists the calculated values ${ }^{8}$ of $I^{*}(0), Q^{*}$, $I^{*}(0) / A^{2}$, and $j(A)$. Given in the last column for comparison are several values of $j(A)$ for the rigidsphere model, as calculated from the phases of Massey and Mohr. ${ }^{3 \mathrm{a}}$ From the present results for the L-J $(12,6)$ potential it appears that Eq. (10) does not adequately describe the relation between $I^{*}(0)$ and $Q^{*}$. The velocity dependence of $Q^{*}$ is discussed later.

\section{BORN APPROXIMATION FOR THE PHASES (L.-J. POTENTIAL)}

For any L-J potential of the form

$$
V(r)=\left(a / r^{q}\right)-\left(b / r^{s}\right),
$$

the Born approximation for the higher order phases may readily be evaluated by an obvious extension of the treatment of Massey and Mohr. ${ }^{3 b}$ The result of the integration is simply

$$
\eta=\eta^{(s)}+\eta^{(q)}
$$

where the phases $\eta^{(s)}$ and $\eta^{(q)}$ are obtained from Eq. (39) of footnote $3 \mathrm{~b}$.

For the L-J $(12,6)$ potential, using symbols concordant with those of footnote $3 b$,

$$
\alpha V=-\left(\frac{C^{(6)}}{r^{6}}+\frac{C^{(12)}}{r^{12}}\right)
$$

where

and

$$
\begin{gathered}
\alpha=2 \mu / \hbar^{2}, \\
C^{(6)}=4 \epsilon \sigma^{6} \alpha=4 B \sigma^{4},
\end{gathered}
$$

$$
C^{(12)}=-4 \epsilon \sigma^{12} \alpha=-4 B \sigma^{10} \text {. }
$$

\& The calculated values of $I(O)$ and $O$ are somewhat sensitive to the cutoff phase (number of higher order phases used). Sample calculations indicate that $j(A)$ may be uncertain by a few percent. 


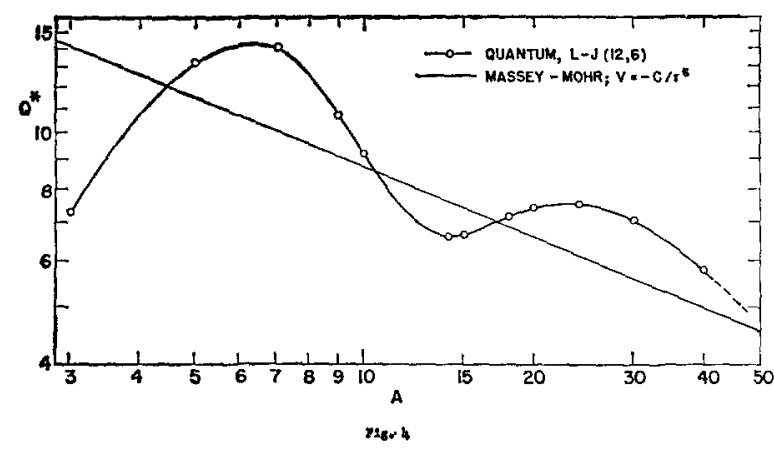

FIG. 4. Velocity dependence of total cross section. $Q^{*} \equiv Q / \pi \sigma^{2}$. $B=125$.

For large $l$ (replacing $l+\frac{1}{2}$ by $l$ ) one obtains the useful approximation formulas for the phases

$$
\begin{aligned}
\eta^{(6)}=(3 \pi / 8) B A^{4} / l^{5} & \text { (attractive), } \\
\eta^{(12)}=-(63 \pi / 256) B A^{10} / l^{11} & \text { (repulsive). }
\end{aligned}
$$

A quantity of interest is the ratio

$$
-\eta^{(12)} / \eta^{(6)}=0.656(A / l)^{6} .
$$

Thus for $l \geqslant 2 A$ the repulsive contribution to the Born phase is $<1 \%$ of the attractive term. As mentioned in I, for the example investigated, Eq. (17) reproduced accurately $( \pm 0.02)$ all directly calculated higher order phases (i.e., for $l \geqslant 2 A$ and $\eta \leqslant 0.5$ ).

\section{APPLICABILITY OF THE MASSEY-MOHR EQUATION FOR THE TOTAL CROSS SECTION}

Massey and $\mathrm{Mohr}^{3 \mathrm{~b}}$ derived a simple formula for the total cross section $Q$ for the potential $\alpha V=-C / r^{s}$, making use of the Born approximation for the higher order phases and utilizing the essentially random character of the lower order phases (mentioned previously). For $s=6$ the Massey-Mohr equation may be written in the following equivalent forms, ${ }^{9}$

$Q=5.720(C / k)^{2 / 5}=5.720 \sigma^{2}(4 B / A)^{2 / 5}$

where

$$
=4.662 \times 10^{11}\left(C^{\prime} / v\right)^{2 / 5},
$$

$$
C^{\prime}=C / \alpha=4 \epsilon \sigma^{6} .
$$

In terms of $Q^{*} \equiv Q / \pi \sigma^{2}$, one obtains a "reduced" form of Eq. (20)

$$
Q^{*}=3.170(B / A)^{2 / 5} \text {. }
$$

It is of interest to investigate the influence of the repulsive part of the potential upon $Q$. Figure 4 shows data taken from Table I [the quantum calculations for the L-J $(12,6)$ potential] plotted in the form $\log Q^{*}$ vs $\log A$. For comparison the appropriate straight line corresponding to Eq. (21) is shown.

Significant undulating deviations are noted (which are attributed to the repulsive contribution to the

${ }^{9}$ E. W. Rothe and R. B. Bernstein, J. Chem. Phys. 31, 1619 (1959). potential). This effect is thought to be a consequence of the broad maximum in the $\eta(l)$ curve (see Fig. 6 of $\mathrm{I}$ ) which, in turn, originates from the negative repulsive phases at low $l$. Although the sign of $\eta$ in itself does not a ffect $\sin ^{2} \eta$ (and thus $Q$ ) the existence of the maximum in $\eta(l)$ gives rise to a certain concentration of nonrandom phases near the maximum. These phases (depending on whether $\sin ^{2} \eta>\frac{1}{2}$ or $<\frac{1}{2}$ ) either add to or subtract from the $Q$ calculated for random phases. The directions of the deviations in Fig. 4 seem to be fairly well accounted for on the basis of these considerations.

The deviations are expected to diminish in magnitude with increasing $A$, as the fraction of the total number of (non-Born) phases which are nonrandom decreases. Thus the velocity dependence of the cross section should approach $v^{-2 / 5}$ as the "semiclassical"10 conditions are achieved, as appears to be the case experimentally. ${ }^{11,12 a}$ However, deviations similar to those of Fig.

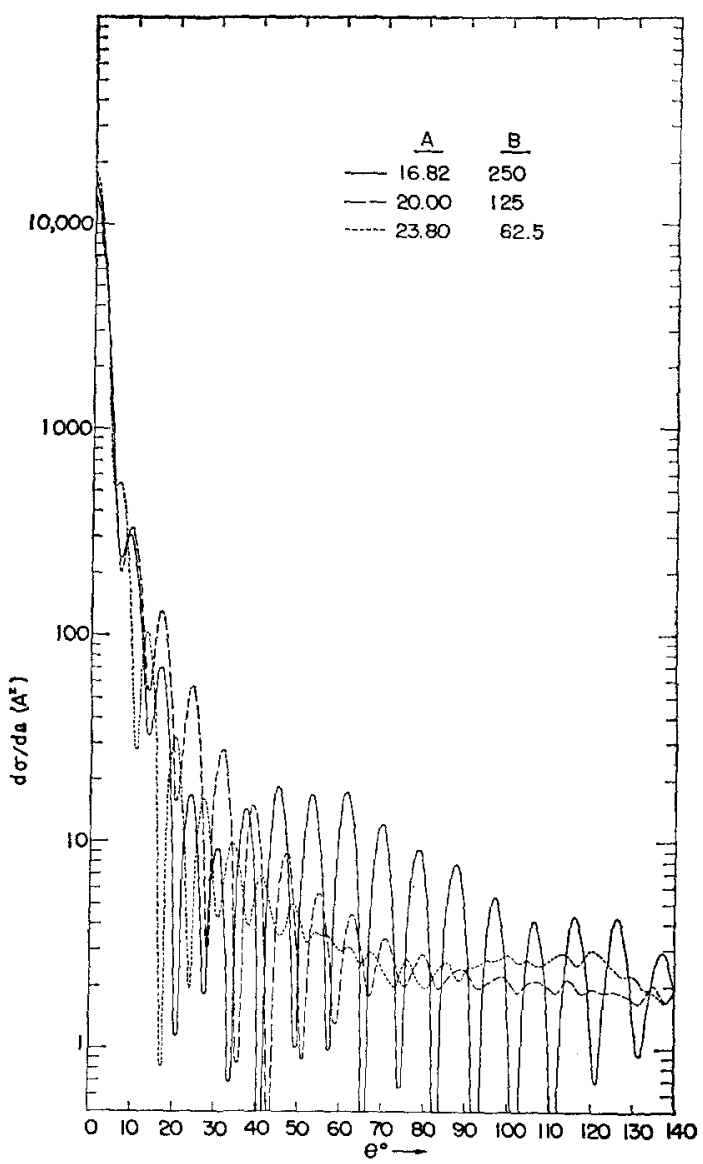

Fig. 5. Influence of $A$ and $B$ (at constant $B A^{4}$ ) upon the angular distribution.

${ }^{10}$ K. W. Ford and J. A. Wheeler, Ann. Phys. (N. Y.) 7, 259, $287(1959)$.

11 H. Pauly, Z. Naturforsch. 15a, 277 (1960).

12 (a) H. Schumacher, R. B. Bernstein, and E. W. Rothe, J. Chem. Phys. 33, 584 (1960). (b) Observations of quantum effects in the scattering of $\mathrm{Li}$ by $\mathrm{Hg}$ have been reported [H. U. Hostettler and R. B. Bernstein, Phys. Rev. Letters 5, 318 (1960)]. 
4 should be observable ${ }^{12 b}$ for beams of the lighter gases at low temperatures.

In the very high velocity region (large $A$ and $K$ ), nearly all phases would be negative; the velocity dependence of $Q$ should (eventually) change from $v^{-2 / 5}$ to $v^{-2 / 11}$. This important consideration has not been treated quantitatively in the present study.

\section{SENSITIVITY OF THE SCATTERING TO THE REPULSIVE PART OF THE POTENTIAL}

Studies of the scattering of thermal velocity molecular beams are expected ${ }^{9,13,14}$ to yield information primarily on the long-range attractive part of the intermolecular potential (via the constant $C^{\prime} \equiv 4 \epsilon \sigma^{6}$ ). However, in view of the discussion of the previous section it is of importance to ascertain the sensitivity of the scattering to the repulsive part.

According to the Massey-Mohr equation, for a given relative velocity or $k$ the total cross section should be independent of $\epsilon$ and $\sigma$ provided $C^{\prime}$ is held constant. In the present notation this is equivalent to the condition

$$
B A^{4}=\text { const }
$$

(at constant $k$, of course). Equation (22) implies [through Eq. (17)] also that all the Born attractive phases will remain unchanged.

A set of calculations was carried out to investigate the effect (on both $d \sigma / d \Omega$ and $Q$ ) of varying $A$ and $B$ at constant $k$, maintaining $B A^{4}=2.00 \times 10^{-7}$. Table II lists the results for $I(0)$ and $Q$. The parameters were chosen to avoid the "bounded region" discussed in I,

\footnotetext{
${ }^{13}$ H. S. W. Massey and R. A. Buckingham, Nature 138, 77 (1936).

${ }^{14}$ H. U. Hostettler and R. B. Bernstein, J. Chem. Phys. 31, $1422(1959)$.
}

TABLE II. Influence of $A$ and $B$ (at constant $B A^{4}$ ) upon $I(0)$ and $Q$.

\begin{tabular}{cccccc}
\hline$K$ & $A$ & $B$ & $\sigma(\mathrm{A})$ & $10^{-3} \times I(0)\left(\mathrm{A}^{2}\right)$ & $Q\left(\mathrm{~A}^{2}\right)$ \\
\hline $1.13^{\mathrm{a}}$ & 16.82 & 250 & 2.447 & 15.7 & 207 \\
$3.20^{\mathrm{b}}$ & 20.00 & 125 & 2.910 & 13.8 & 198 \\
$9.06^{\mathrm{a}}$ & 23.80 & 62.5 & 3.460 & 18.2 & 184 \\
\hline
\end{tabular}

a Phases estimated using $Q-i-K$ curves from I (extended).

b Phases from Table III of I (extended).

enabling the use of the $Q-i-K$ curves (the "reduced phase" treatment) to estimate the phases for $K=1.13$ and 9.06. The Massey-Mohr equation (20) yields $Q=$ $176 \mathrm{~A}^{2}$ (for the three cases).

Figure 5 shows a comparison of the three calculated angular distributions. Although the curves are easily distinguishable, the problem of deriving a unique or "best" set of parameters $\epsilon, \sigma$ for a given observed angular distribution (i.e., the "inversion" problem) appears to be a formidable one indeed.

One may conclude that while the influence of the repulsive part of the potential is important (and should be observable under certain conditions), the attractive term dominates in determining the scattering of thermal velocity molecular beams.

\section{ACKNOWLEDGMENTS}

The author wishes to express his appreciation to Professor P. J. W. Debye (Senior Scientist, Institute of Science and Technology, University of Michigan) for a number of valuable discussions and suggestions. The assistance of Mrs. H. Schumacher in plotting graphs and arranging tables is acknowledged with thanks. 